\title{
Absorption from Neutral Acceptors in GaAs and GaP
}

\section{Christensen, Ove}

\section{Published in:}

Physical Review B

Link to article, DOI:

10.1103/PhysRevB.7.1426

Publication date:

1973

\section{Document Version}

Publisher's PDF, also known as Version of record

Link back to DTU Orbit

Citation (APA):

Christensen, O. (1973). Absorption from Neutral Acceptors in GaAs and GaP. Physical Review B, 7(4), 14261432. https://doi.org/10.1103/PhysRevB.7.1426

\section{General rights}

Copyright and moral rights for the publications made accessible in the public portal are retained by the authors and/or other copyright owners and it is a condition of accessing publications that users recognise and abide by the legal requirements associated with these rights.

- Users may download and print one copy of any publication from the public portal for the purpose of private study or research.

- You may not further distribute the material or use it for any profit-making activity or commercial gain

- You may freely distribute the URL identifying the publication in the public portal

If you believe that this document breaches copyright please contact us providing details, and we will remove access to the work immediately and investigate your claim 
with an without the magnetic field. The fact that the rates differed in the experiment indicates that in the strong flux regime, the theory presented here must be modified.
${ }^{1}$ E. M. Conwell and A. K. Ganguly, Phys. Rev。B 4 , 2535 (1971).

${ }^{2}$ M. C. Steele, RCA Rev. 28, 58 (1967).

${ }^{3} \mathrm{H}$. Hayakawa and M. Kikuchi, Appl. Phys. Letters 12, 251 (1968).

${ }^{4}$ E. Voges, Solid State Commun. $\underline{8}, 1733$ (1970).
${ }^{5}$ H. N. Spector, Phys. Rev. B 6, 2409 (1972).

${ }^{6}$ Handbook of Mathematical Functions, edited by M. Abramowitz and I. A. Stegun, NBS Appl. Math. Series 55 (U. S. GPO, Washington, D. C., 1964), p. 374.

${ }^{7}$ T. E. Parker and R. Bray, Bull. Am. Phys. Soc. 17, 370 (1972).

\title{
Absorption from Neutral Acceptors in GaAs and GaP
}

\author{
Ove Christensen* \\ Physics Laboratory III, Technical University of Denmark, Lyngby, Denmark \\ (Received 17 April 1972)
}

\begin{abstract}
We present a new calculation of the absorption due to transitions of holes between neutral acceptors and the various valence-band sublevels in GaAs and GaP. The acceptor wave function was approximated by a previously suggested expression for ground-state wave functions appropriate to complicated band extrema. Numerical calculations of the absorption from intervalence-band transitions of free holes and neutral acceptors have been performed. Good agreement with experimental results is obtained.
\end{abstract}

\section{INTRODUCTION}

Optical transitions of free holes between pairs of the three sublevels of the valence band in GaAs and $\mathrm{GaP}$ give rise to a characteristic infrared absorption. ${ }^{1-3}$ In GaAs at room temperature, the absorption has qualitatively the same shape as the absorption due to the same transitions among the valence-band levels of $\mathrm{Ge}^{2,4-7}$ Balslev $^{2}$ calculated the room-temperature absorption in $p$ type GaAs and obtained good agreement with experimental results. No interpretation of the $a b-$ sorption at $77^{\circ} \mathrm{K}^{1}$ has been published. Recently, Wiley and DiDomenico (WD) ${ }^{3}$ measured the infrared absorption of $p$-type GaP. In contrast to GaAs, the absorption spectrum of GaP has no significant structure. WD made a calculation of the room-temperature absorption, based on the model of $\mathrm{Kahn}^{4}$ for inter-valence-band transitions. To get agreement with experimental results it was necessary to use two adjustable parameters plus a contribution from intraband free-carrier absorption. At $90^{\circ} \mathrm{K}$, WD identified the absorption as being due to neutral acceptors. No calculation of this absorption was made.

In this work, we present a model for absorption from neutral acceptors with applications to GaAs and GaP. The basic absorption mechanism is that of transitions of holes from the acceptor state to the sublevels of the valence band. In Sec. II we develop the necessary theory for absorption due to such transitions. Analytical calculations based on a simple band structure are performed and a comparison is made with the absorption from inter-valence-band transitions of free holes. In order to perform numerical calculations on GaAs and GaP we make approximations for the acceptor wave function. Here, we use a previously analyzed form for the ground-state wave function. ${ }^{8}$ In Sec. III we give a description of the computation of the absorption in a $\operatorname{model}^{2,7}$ which takes into account the detailed structure of the valence band and the optical matrix elements. In Sec. IV we present experimental results for the absorption of $p$-type GaAs together with the experimental results of WD for $p$-type GaP. The experimental data are compared with computed spectra for inter-valence-band absorption and for absorption from neutral acceptors.

\section{ABSORPTION FROM NEUTRAL ACCEPTORS}

\section{A. Theory}

At a sufficiently low temperature, the free holes in a semiconductor return to the acceptor ions and then neutralize these negatively charged states. The hole is in other words bound to the negative acceptor center. Let the valence-band Bloch functions with wave vector $\overrightarrow{\mathrm{k}}$ be given by $\phi_{i, \overrightarrow{\mathrm{k}}}(\overrightarrow{\mathrm{r}})$ $=u_{i \vec{k}}(\overrightarrow{\mathrm{r}}) e^{i \overrightarrow{\mathrm{k}} \cdot \overrightarrow{\mathrm{r}}}$, where $i=1,2$, and 3 denote the heavyhole band, light-hole band, and spin-orbit splitoff band, respectively. $u_{i, \overrightarrow{\mathrm{k}}}(\overrightarrow{\mathrm{r}})$ are periodic func- 
tions of position $\overrightarrow{\mathbf{r}}$ with the periodicity of the lattice. The wave function $\psi(\overrightarrow{\mathrm{r}})$ of the bound hole can be expanded in terms of the Bloch functions of the valence bands around $\overrightarrow{\mathrm{k}}=0$ :

$$
\psi(\overrightarrow{\mathrm{r}})=\sum_{i, \overrightarrow{\mathrm{r}}} a_{i}(\overrightarrow{\mathrm{k}}) \phi_{i, \overrightarrow{\mathrm{k}}}(\overrightarrow{\mathrm{r}}),
$$

where the summation extends over all quantum states $\vec{k}$. We shall here only be concerned with the case where all the carriers occupy the acceptor ground state of energy $E_{a}$.

The physical absorption mechanism consists of optical excitation of bound holes to free-carrier states in the valence-band levels. Let index $a$ denote the acceptor state. The optical matrix element for a transition from the acceptor state to a free-hole state at wave vector $\vec{k}$ in valence band $j$ is given by

$$
M_{a j}(\overrightarrow{\mathrm{k}})=\left\langle\phi_{j, \overrightarrow{\mathrm{k}}}(\overrightarrow{\mathrm{r}})|\overrightarrow{\mathrm{e}} \cdot \overrightarrow{\mathrm{p}}| \psi(\overrightarrow{\mathrm{r}})\right\rangle,
$$

where $\overrightarrow{\mathrm{e}}$ is a unit vector in the direction of the polarization of the incident light. The absorption constant due to transitions to band $j$ is then ${ }^{9}$

$$
\begin{aligned}
\alpha_{a j}=\left(N_{a} / n\right)(C / \hbar \omega) \int d^{3} \overrightarrow{\mathrm{k}}\left|M_{a j}\right|^{2}\left(f_{a}-f_{j}\right) \\
\times \delta\left(E_{a}-\epsilon_{j}(\overrightarrow{\mathrm{k}})-\hbar \omega\right),
\end{aligned}
$$

where $N_{a}$ is the concentration of neutral acceptors, $C$ a constant, and $n$ is the refractive index. $f_{a}$ and $f_{j}$ are normalized occupation probabilities for holes with energies $E_{a}$ and $\epsilon_{j}(\overrightarrow{\mathrm{k}})$, respectively. At the low temperatures of interest here $f_{a} \simeq 1$ and $f_{j} \simeq 0$. Inserting the expression (1) the matrix element (2) can be written

$$
M_{a j}=\sum_{i} a_{i}(\overrightarrow{\mathrm{k}}) M_{i j}(\overrightarrow{\mathrm{k}}) \text {. }
$$

$M_{i j}$ is the optical matrix element ${ }^{4,14}$ for transitions between the valence band levels $i$ and $j$,

$$
M_{i j}(\overrightarrow{\mathrm{k}})=\left\langle\phi_{j, \overrightarrow{\mathrm{k}}}(\overrightarrow{\mathrm{r}})|\overrightarrow{\mathrm{e}} \cdot \overrightarrow{\mathrm{p}}| \phi_{i, \overrightarrow{\mathrm{k}}}(\overrightarrow{\mathrm{r}})\right\rangle .
$$

Using Eq. (4) the expression (3) for $\alpha_{a j}$ can be written

$$
\begin{aligned}
\alpha_{a j}=\left(N_{a} / n\right)(C / \hbar \omega) \int d^{3} \overrightarrow{\mathrm{k}} & \left|\sum_{i} a_{i}(\overrightarrow{\mathrm{k}}) M_{i j}\right|^{2} \\
& \times \delta\left(E_{a}-\epsilon_{j}(\overrightarrow{\mathrm{k}})-\hbar \omega\right) .
\end{aligned}
$$

It is useful to compare this expression with the absorption arising from transitions of free holes $2,4,7,14$ of concentration $p$ between bands $i$ and $j$ :

$\alpha_{i j}=(p C / n \hbar \omega) \int d^{3} \overrightarrow{\mathrm{k}}\left|M_{i j}\right|^{2}\left(f_{i}-f_{j}\right) \delta\left(\epsilon_{i}(\overrightarrow{\mathrm{k}})-\epsilon_{j}(\overrightarrow{\mathrm{k}})-\hbar \omega\right)$,

where for nondegenerate statistics at temperature $T, f_{i} \propto\left(k_{B} T\right)^{-3 / 2} e^{\epsilon_{i}(\overrightarrow{\mathrm{k}}) / k_{B} T}\left[\epsilon_{i}(\overrightarrow{\mathrm{k}}) \leq 0\right]$. Here, $k_{B}$ is Boltzmann's constant. In Eq. (6) normalization of the wave function (1) requires $\sum_{i, \overrightarrow{\mathrm{k}}}\left|a_{i}(\overrightarrow{\mathrm{k}})\right|^{2}=1$, which in Eq. (7) is replaced by $\sum_{i, \overrightarrow{\mathrm{k}}} f_{i}\left(\epsilon_{i}(\overrightarrow{\mathrm{k}})\right)=1$.

The absorption due to transitions from a localized state to free-carrier states in the valence bands has then been broken up into a sum over mo- mentum-conserving transitions between the valenceband levels. The absorption from neutral acceptors is then expressed by the same quantities as determine the inter-valence-band absorption, the thermal occupation probability $f_{i}$ in Eq. (7) essentially being replaced by the probability amplitude $\left|a_{i}\right|^{2}$ in Eq. (6). $\alpha_{i j}$ and $\alpha_{a j}$ can, therefore, be computed numerically by the same procedure. This is particularly convenient since the computation of intervalence-band absorption can be performed by very accurate methods. ${ }^{2,7}$

\section{B. Model Based on Simple Band Structure}

In order to investigate the main features of the absorption from neutral acceptors, we shall assume spherical heavy-hole and split-off bands of masses $m_{1}$ and $m_{3}$, respectively. The $\overrightarrow{\mathrm{k}}=0$ separation between these bands is $\Delta$, the spin-orbit splitting, which is assumed much larger than the acceptor binding energy. We furthermore assume that the light-hole mass is much smaller than the heavyhole mass. The coupling between the acceptor state and the split-off and light-hole bands will then be neglected such that

$$
\psi(\overrightarrow{\mathrm{r}}) \simeq \sum_{\overrightarrow{\mathrm{k}}} a_{1}(\overrightarrow{\mathbf{k}}) \phi_{1, \overrightarrow{\mathrm{k}}}(\overrightarrow{\mathrm{r}}) .
$$

For a hydrogenic ground state $a_{1}(\overrightarrow{\mathrm{k}})$ is the Fourier transform of the ground-state hydrogenlike wave function $^{10}$ :

$$
a_{1}(\overrightarrow{\mathrm{k}})=8 \sqrt{\pi} \lambda^{5 / 2} /\left(\lambda^{2}+k^{2}\right)^{2},
$$

where $\lambda=1 / a_{H}, a_{H}$ being the Bohr radius of the problem. The acceptor binding energy is given by $E_{a}=\left(\hbar^{2} / 2 m_{1}\right) \lambda^{2}$, and Eq. (8) can then be written

$$
a_{1}(\overrightarrow{\mathrm{k}}) \propto\left[E_{a}-\epsilon_{1}(\overrightarrow{\mathrm{k}})\right]^{-2} \text {. }
$$

At photon energies larger than the spin-orbit splitting, the main part of the absorption is due to optical excitation of carriers in the acceptor state to the split-off band, $a-3$ transitions. Using ${ }^{4}\left|M_{i j}\right|^{2}$ $\propto k^{2}$ together with the above-mentioned approximation, we find

$$
\begin{aligned}
\alpha_{a 3}=K \frac{16}{\pi} \frac{N_{a}}{n \hbar \omega} & \left(E_{a}\right)^{-3 / 2}\left[\hbar \omega-\left(\Delta+E_{a}\right)\right]^{3 / 2} \\
& \times\left(1+\frac{m_{1}}{m_{3}} \frac{\hbar \omega-\left(\Delta+E_{a}\right)}{E_{a}}\right)^{-4},
\end{aligned}
$$

where $K$ is a constant. The corresponding expression for 1-3 transitions of free holes is given by $\mathrm{Kahn}^{4}$ :

$$
\begin{aligned}
\alpha_{13}=K \frac{2}{\sqrt{\pi}} \frac{p}{n \hbar \omega} & \left(k_{B} T\right)^{-3 / 2}(\hbar \omega-\Delta)^{3 / 2} \\
& \times \exp \left(-\frac{m_{3}}{m_{1}-m_{3}} \frac{\hbar \omega-\Delta}{k_{B} T}\right) .
\end{aligned}
$$

In these expressions, the increase of absorption close to $\hbar \omega \simeq \Delta$ is due to the influence of density of states and optical matrix elements. The behavior 
at high photon energies is dominated by the probability amplitude $\left|a_{1}\right|^{2}$ for $\alpha_{a 3}$ and by the thermal occupation probability for $\alpha_{13}$. The threshold energy for $a-3$ transitions is $\Delta+E_{a}$ compared to $\Delta$ for 1- 3 transitions.

The maximum of the absorption occurs at photon energies of

$$
\begin{aligned}
& \hbar \omega_{13}^{\max } \simeq \Delta+\frac{3}{2} k_{B} T\left[\left(m_{1}-m_{3}\right) / m_{3}\right], \\
& \hbar \omega_{a 3}^{\max } \simeq \Delta+E_{a}\left[1+\frac{3}{5}\left(m_{1} / m_{3}\right)\right] .
\end{aligned}
$$

For $N_{a}=p$ the ratios between the maximum absorption cross sections is

$$
\alpha_{a 3}^{\max } / \alpha_{13}^{\max } \simeq 0.80 \times\left[m_{1} /\left(m_{1}-m_{3}\right)\right]^{3 / 2} .
$$

In most materials, the ratio $\alpha_{a 3}^{\max } / \alpha_{13}^{\max }$ is larger than unity. (GaAs:1.20; GaP:1.40). The maximum absorption will then increase for decreasing temperatures where more holes are bound to acceptor sites.

Within this simple theory, the absorption from neutral acceptors thus provides information about the acceptor binding energy from the position of the maximum absorption [Eq. (12)]. Owing to the one-to-one correspondence between photon energy and wave vector the acceptor wave function, given by $a_{1}(\overrightarrow{\mathrm{k}})$, can be found.

\section{Approximations for $\mathrm{GaAs}$ and $\mathrm{GaP}$}

The actual valence-band structure of GaAs and GaP is complicated by warping and by nonparabolicity. We shall first consider the problem of determining the Fourier components $a_{1}(\overrightarrow{\mathrm{k}})$ and $a_{2}(\overrightarrow{\mathrm{k}})$ of the bound-hole wave function (1). For a carrier with complicated effective mass bound by a Coulomb potential, we have previously ${ }^{8}$ suggested the approximate wave function

$$
a_{i}(\overrightarrow{\mathrm{k}})=Q_{i}\left[E_{a}-\epsilon_{i}(\overrightarrow{\mathrm{k}})\right]^{-\alpha}\left[E_{a}-\epsilon(\overrightarrow{\mathrm{k}})\right]^{\alpha-2}
$$

where $Q_{i}$ is a normalization constant, $\epsilon_{i}(\overrightarrow{\mathrm{k}})$ $=-\hbar^{2} k^{2} / 2 m_{i}, m_{i}$ being the average $\overrightarrow{\mathrm{k}}=0$ mass of band $i . \quad E_{a}$ is the actual experimental value of the acceptor binding energy. $Q_{i}$ are normalization constants to be discussed in connection with the actual band structure and $q$ is an anisotropy parameter. This result was derived from an iterative procedure, based on the effective-mass equation in the momentum representation. For a band with energy given by $\epsilon(\vec{k})[\epsilon(\vec{k})<0]$ this equation is 8,11

$$
\left[\epsilon(\overrightarrow{\mathrm{k}})-E_{a}\right] a(\overrightarrow{\mathrm{k}})+\int d^{3} \overrightarrow{\mathrm{k}}^{\prime} a\left(\overrightarrow{\mathrm{k}}^{\prime}\right) U\left(\overrightarrow{\mathrm{k}}-\overrightarrow{\mathrm{k}}^{\prime}\right),
$$

where $U(\vec{k})$ is the Fourier transform of the impurity potential. The form (15) is very suitable for iterative methods since a trial function $a^{0}(\overrightarrow{\mathrm{k}})$ inserted in the integral produces a new function

$$
a^{1}(\overrightarrow{\mathrm{k}})=\frac{1}{E_{a}-\epsilon(\overrightarrow{\mathrm{k}})} \int d^{3} \overrightarrow{\mathrm{k}}^{\prime} a^{0}\left(\overrightarrow{\mathrm{k}}^{\prime}\right) U\left(\overrightarrow{\mathrm{k}}-\overrightarrow{\mathrm{k}}^{\prime}\right),
$$

which is better than $a^{0}(\overrightarrow{\mathrm{k}})$. In Eq. (14), $q=2$ is the zero-order trial function, obtained from the case of spherical bands [Eq. (9)]. The first iteration yields the wave function with $q=1$. The formula (14) was examined ${ }^{8}$ for ellipsoidal, parabolic bands and for nonparabolic, isotropic bands in the range $0 \leq q \leq 1$. The best result for the expectation value of the ground-state energy was obtained for $q \simeq 0.5$.

Figures 1 and 2 show the valence-band structure of GaAs and GaP as calculated from the fourband $\overrightarrow{\mathrm{k}} \cdot \overrightarrow{\mathrm{p}}$ model described in Sec. III. The parabolic behavior of the heavy-hole bands is only present in the $\langle 100\rangle$ and $\langle 111\rangle$ directions. In other directions, small deviations from parabolicity occur. The average $\overrightarrow{\mathrm{k}}=0$ masses are displayed in Table I. The coupling from the acceptor to the valence bands is dependent on the hole energy compared to the acceptor energy. Owing to the energy denominator in Eq. (14) we shall neglect the admixture of split-off band states in the acceptor wave function (1). For GaAs, the lighthole band can be considered as a remote band except in the region close to $\vec{k}=0$. This region contributes, however, little to the absorption since the optical matrix elements depend on the square of the wave vector. The contribution from light-hole states in Eq. (1) will therefore be neglected. The matrix elements of Eq. (4) are in this approximation

$$
M_{a 1} \simeq a_{1} M_{11}, \quad M_{a 2} \simeq a_{1} M_{12}, \quad M_{a 3} \simeq a_{1} M_{13},
$$
with

$$
\sum_{\overrightarrow{\mathrm{k}}}\left|a_{1}(\overrightarrow{\mathrm{k}})\right|^{2}=1
$$

For GaP the heavy - and light-hole band are

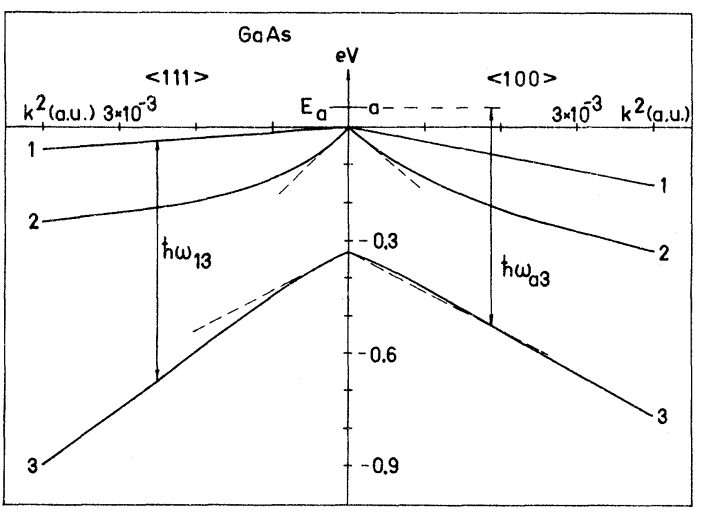

FIG. 1. Valence-band structure of GaAs at $300^{\circ} \mathrm{K}$ in the $\langle 100\rangle$ and $\langle 111\rangle$ directions ( 1 a.u. $\left.=1 / a_{0}\right)$. Full line: calculated by the four-band $\vec{k} \cdot \vec{p}$ model described in Sec. III. Broken line: $\overrightarrow{\mathrm{k}}=0$ slopes. Left-hand side illustrates 1-3 transitions. Right-hand side illustrates transitions of bound holes from the acceptor level to the split-off band. 


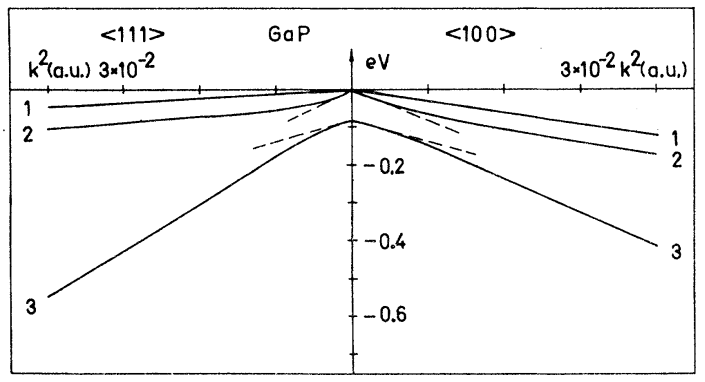

FIG. 2. Valence-band structure of GaP in the $\langle 100\rangle$ and $\langle 111\rangle$ directions. Full line: calculated from fourband $\vec{k} \cdot \vec{p}$ model. Broken line: $\vec{k}=0$ slopes.

parallel over most of the region in $\vec{k}$ space which contributes significantly to the absorption. The asymptotic distance between the bands is $\frac{2}{3} \Delta$ $=55 \mathrm{meV}$. This distance can over a large part of $\overrightarrow{\mathrm{k}}$ space be considered as small compared to the energy separation between the acceptor state and the two bands. Asymptotically, we must thus have $\left|a_{1}\right|=\left|a_{2}\right|$ yielding $\left|Q_{1}\right| \simeq\left|Q_{2}\right|$ at large wave vectors. We are not able to determine the phase of $a_{1}$ and $a_{2}$ and in Eq. (6) we must, therefore, make the approximation

$$
\left|\sum_{i} a_{i} M_{i j}\right|^{2} \simeq \sum_{i}\left|a_{i}\right|^{2}\left|M_{i j}\right|^{2} \text {. }
$$

The matrix elements $M_{a j}$ in Eq. (4) are thus given by

$$
\begin{aligned}
& \left|M_{a 1}\right|^{2}=\left|a_{1}\right|^{2}\left|M_{11}\right|^{2}+\left|a_{2}\right|^{2}\left|M_{12}\right|^{2}, \\
& \left|M_{a 2}\right|^{2}=\left.\left|a_{1}\right|^{2} M_{21}\right|^{2}+\left|a_{2}\right|^{2}\left|M_{22}\right|^{2}, \\
& \left|M_{a 3}\right|^{2}=\left|a_{1}\right|^{2}\left|M_{13}\right|^{2}+\left|a_{2}\right|^{2}\left|M_{23}\right|^{2},
\end{aligned}
$$

with

$$
\sum_{\overrightarrow{\mathrm{k}}}\left|a_{1}\right|^{2}+\left|a_{2}\right|^{2}=1
$$

\section{COMPUTATION OF ABSORPTION CONSTANT}

A procedure for numerical computation of the absorption constant has been set up. Except for minor details, the calculation was performed as described by Arthus, Baynham, Fawcett, and Paige ${ }^{7}$ for the absorption from inter-valenceband transitions in Ge. In our $\overrightarrow{\mathrm{k}} \cdot \overrightarrow{\mathrm{p}}$ treatment of the energy levels, we have taken into account the twofold-degenerate Kramers doublets of the three $\Gamma_{15}$ valence-band levels and the $\overrightarrow{\mathrm{k}}=0$ conductionband state $\Gamma_{1}$. The energies of these states were found by diagonalizing $8 \times 8$ Hermitian matrices. The $\overrightarrow{\mathrm{k}} \cdot \overrightarrow{\mathrm{p}}$-matrix elements were the $k$ linear terms between the valence bands and the conduction band ${ }^{12}$ and $k^{2}$ elements ${ }^{13,14}$ giving the interaction between the valence-band levels through intermediate states, except the $\overrightarrow{\mathrm{k}}=0$ conduction-band level. Our $\overrightarrow{\mathrm{k}} \cdot \overrightarrow{\mathrm{p}}$ model is similar to the one used by Balslev ${ }^{2}$ but no approximations were made in the present calculation. The absorption spectrum was obtained in the form of a histogram. A Gaussian broadening ${ }^{15}$ was applied to the histogram. The standard deviation of the broadening was 10 meV at 77 and $90{ }^{\circ} \mathrm{K}$ and $15 \mathrm{meV}$ at $300^{\circ} \mathrm{K}$. The fluctuations in the absorption spectrum thus obtained were typically 5\%. The absorption from free holes was calculated by taking into account all kinds of inter-valence-band transitions, i.e., 1-2, 2-3, and 1-3 transitions. In the calculation of the absorption from bound holes, Eq. (6) was used with the approximations for the matrix elements discussed in Sec. IIC.

Calculations were performed for GaAs both with the experimentally determined valence-band parameters of Balslev ${ }^{2}$ and with the calculated ones of Lawaetz. ${ }^{16}$ The latter gave $10 \%$ larger absorption cross section than the former but apart from this, no deviations were found. In the calculation on GaP, the valence-band parameters of Lawaetz ${ }^{16}$ were used. Except for the acceptor binding energy and the anisotropy parameter $q$, no adjustable parameters were used. The latter was varied in the interval $0 \leq q \leq 1$, which only produced a rather small effect. It should be noted that the absolute value of the absorption constant was calculated. The parameters used in the calculation are displayed in Table $I$.

\section{COMPARISON WITH EXPERIMENT}

\section{A. Results on GaAs}

Absorption measurements were carried out on $\mathrm{Zn}$-doped GaAs at 300 and $77^{\circ} \mathrm{K}$. The absorption was determined from sample-in-sample-out measurements, using a refractive index of 3.3 . The concentration of free carriers determined from Hall measurements was $1.4 \times 10^{17} \mathrm{~cm}^{-3}$ at $300{ }^{\circ} \mathrm{K}$ and $3.0 \times 10^{16} \mathrm{~cm}^{-3}$ at $77^{\circ} \mathrm{K}$. The concentration of uncompensated acceptors is equal to the carrier concentration at $300^{\circ} \mathrm{K}$. The measured

TABLE I. Valence-band parameters (Ref. 16) of GaAs and GaP.

\begin{tabular}{cccc}
\hline \hline Parameter & GaAs & GaP & Unit \\
\hline$A$ & -7.65 & -4.20 & $\hbar / 2 m_{0}$ \\
$B$ & -4.82 & -1.96 & $\cdots$ \\
$N$ & -19.68 & -9.96 & $\cdots$ \\
$P^{2}$ & 25.7 & 22.2 & $(\mathrm{eV}) \hbar^{2} / 2 m_{0}$ \\
$\left\langle m_{1}\right\rangle$ & 0.62 & 0.79 & $m_{0}$ \\
$\left\langle m_{2}\right\rangle$ & 0.074 & 0.14 & $\cdots$ \\
$\left\langle m_{3}\right\rangle$ & 0.15 & 0.24 & $\cdots$ \\
$E_{0,300}{ }^{\circ} \mathrm{K}$ & $1.43^{\mathrm{a}}$ & $2.78^{\mathrm{b}}$ & $\mathrm{eV}$ \\
$E_{0,77}{ }^{\mathrm{K}}$ & $1.52^{\mathrm{a}}$ & $2.86^{\mathrm{b}}$ & $\cdots$ \\
$\Delta$ & $0.33^{\mathrm{c}}$ & $0.082^{\mathrm{b}}$ & $\cdots$ \\
\hline \hline
\end{tabular}

${ }^{a}$ M. P. Sturge, Phys. Rev. 127, 768 (1963).

${ }^{b}$ P. J. Dean, G. Kaminsky, and R. B. Zetterstrom, J. Appl. Phys。 38, 3551 (1967)。

'Reference 1 . 
absorption at room temperature is shown in Fig. 3 together with the calculated absorption from inter-valence-band transitions of free carriers at this temperature. A constant contribution of $1 \mathrm{~cm}^{-1}$ has been subtracted from all experimental values. Apart from this correction, no adjustable parameters were used. There is excellent agreement between theory and experiment at large photon energies. For $\hbar \omega<0.45 \mathrm{eV}$, there is a significant additional absorption. The experimental absorption cross section at $0.42 \mathrm{eV}$ is $1.3 \times 10^{-16} \mathrm{~cm}^{2}$. Braunstein ${ }^{1}$ found values between 0.65 and $0.9 \times 10^{-16} \mathrm{~cm}^{2}$. The calculated cross section at $0.45 \mathrm{eV}$ is $1.1 \times 10^{-16} \mathrm{~cm}^{2}$ in agreement with Balslev's ${ }^{2}$ result. The room-temperature absorption provides a check on the parameters of the calculation.

In Fig. 4 we show experimental and calculated absorption spectra at $77^{\circ} \mathrm{K}$. The calculated absorption from momentum-conserving transitions of free carriers $\left(3.0 \times 10^{16} \mathrm{~cm}^{-3}\right)$ is seen to deviate much from the experimental results. Assuming absorption due to transitions of bound holes to the split-off band to be predominant, we can estimate the acceptor binding energy from Eq. (12). The interval of photon energy from the spin-orbit splitting to the maximum of absorption is $\simeq 60 \mathrm{meV}$. With the average mass of Table I for the heavyhole band and split-off band, Eq. (12) yields $E_{a}$ $\simeq 18 \mathrm{meV}$. This is in good agreement with recent values ${ }^{17}$ for the binding energy of $\mathrm{Zn}$ acceptors.

The absorption from neutral acceptors of concentration $1.1 \times 10^{17} \mathrm{~cm}^{-3}$ was computed using the approximations (17). The calculation was done for different values of the acceptor binding energy and the anisotropy parameter $q$ of Eq. (14). As expected from Eq. (10) the absorption is strongly

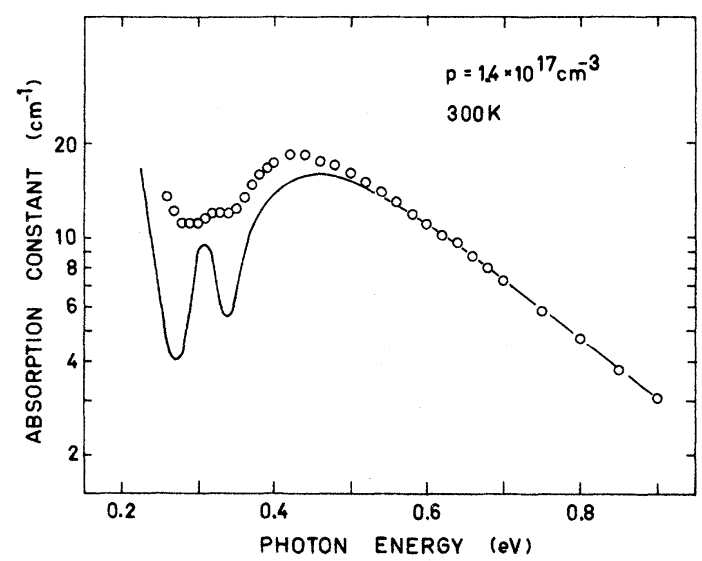

FIG. 3. Absorption constant of $p$-type GaAs at room temperature. Circles: experimental points. Full line: calculated spectrum from inter-valence-band transitions of free holes.

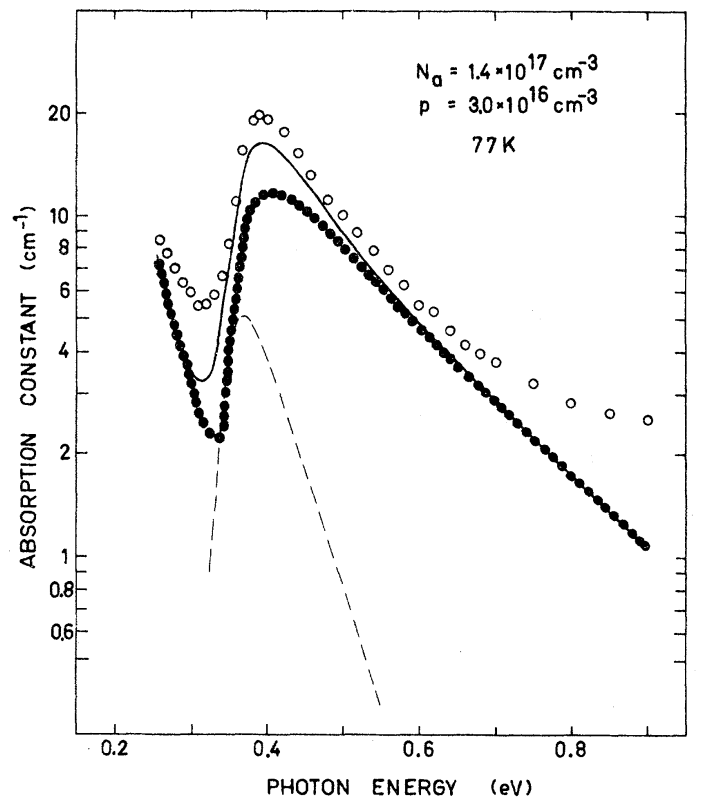

FIG. 4. Absorption constant of $p$-type GaAs at $77^{\circ} \mathrm{K}$. Open ci:cles: experimental points. Broken line: calculated absorption from free-carrier transitions at $77^{\circ} \mathrm{K}$. Filled circles: calculated absorption from neutral acceptors $\left(E_{a}=24 \mathrm{meV}, q=1\right)$. Full line: calculated total contribution from free and bound holes.

dependent on $E_{a}$, the spectrum becoming broader with increasing binding energy. For given $q$, the acceptor binding energy which produced best agreement with experiment could be determined within $10 \%$. The dependence of the absorption on $q$ is rather weak. The best fit to experiment was obtained for $E_{a}=24 \mathrm{meV}$. A change of $q$ from 0 to 1 produced a $20 \%$ larger absorption at $\hbar \omega=0.90$ $\mathrm{eV}$. The absorption has thus no significant dependence of $q$. The dotted line in Fig. 4 shows the calculated absorption from neutral acceptors with $E_{a}=24 \mathrm{meV}, q=1$. The sum of the contributions from bound and free holes is seen to agree well with experimental data.

There is a disagreement between the experimental and calculated absorption around $\hbar \omega \simeq 0.30$ $\mathrm{eV}$ and for $\hbar \omega>0.65 \mathrm{eV}$. These regions correspond to transitions from the acceptor state to the light-hole band and split-off band, respectively (see Fig. 1). Neglecting the nonparabolicity of the split-off band, a transition from the acceptor state to this band with $\hbar \omega=0.65 \mathrm{eV}$ takes place at $k \simeq 5-6 \times 10^{-2}$ a.u. $\left(1\right.$ a. u. $\left.=1 / a_{0}\right)$. Transitions to the light-hole band at this wave vector correspond to photon energies from 0.25 to $0.32 \mathrm{eV}$. The reason for the disagreement is obviously that the strength of the Fourier coefficient $a_{1}(\overrightarrow{\mathrm{k}})$ of Eq. (1) is underestimated at large wave vectors by the hydrogenlike wave function. A typical 
spatial distance connected with $k=6 \times 10^{-2}$ a.u. is $R=\frac{1}{2} \pi /\left(6 \times 10^{-2}\right.$ a. u. $) \simeq 15 \AA$. One would then expect the core potential of the $\mathrm{Zn}$ ion plus the local strain fields to extend that far out. For a deep acceptor level, Lucovsky ${ }^{18}$ found that the excited acceptor states could be described by a longrange Coulombic potential. Accordingly, the core potential would be expected to reach out a distance between the first and second Bohr radius (10 and $40 \AA$ ) as found.

\section{B. Results on GaP}

For GaP, we have used the experimental results of $\mathrm{WD}^{3}$ at 300 and $90^{\circ} \mathrm{K}$. As WD, we shall use wavelength scale instead of photon energy scale. Figure 5 shows the calculated absorption from free carriers at room temperature together with WD's experimental results for their sample $B$ $\left(N_{a}=2.0 \pm 0.2 \times 10^{17} \mathrm{~cm}^{-3}, p=1.6 \pm 0.2 \times 10^{17} \mathrm{~cm}^{-3}\right.$ at $300{ }^{\circ} \mathrm{K}$ ). The calculation was done with $p=1.6$ $\times 10^{17} \mathrm{~cm}^{-3}$. As described by WD, the absorption bands from 1-3 and 2-3 transitions overlap. According to the band structure of Fig. 2, the region of 1-2 transitions occurs at wavelengths larger than $\simeq 24 \mu$. Lattice absorption obscures the spectrum at $\lambda>12 \mu$. In the wavelength range of interest, 1-2 transitions, therefore, do not con-

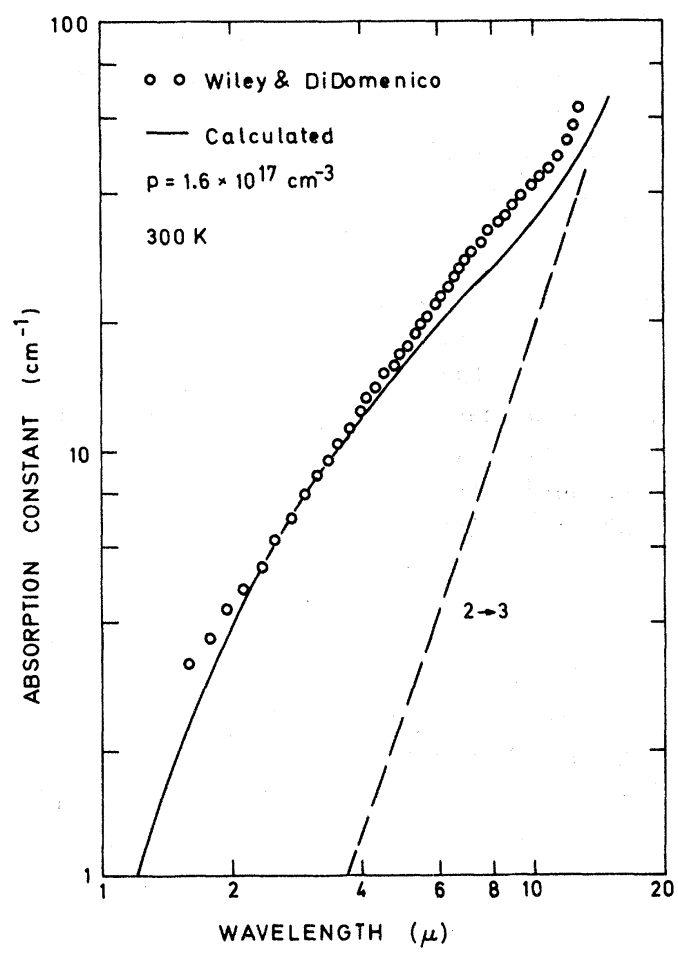

FIG. 5. Absorption constant of $p$-type GaP at room temperature. Circles: experimental results of Ref. 3 . Full line: calculated absorption from free-carrier transitions. Broken line: contribution from 2-3 transitions.

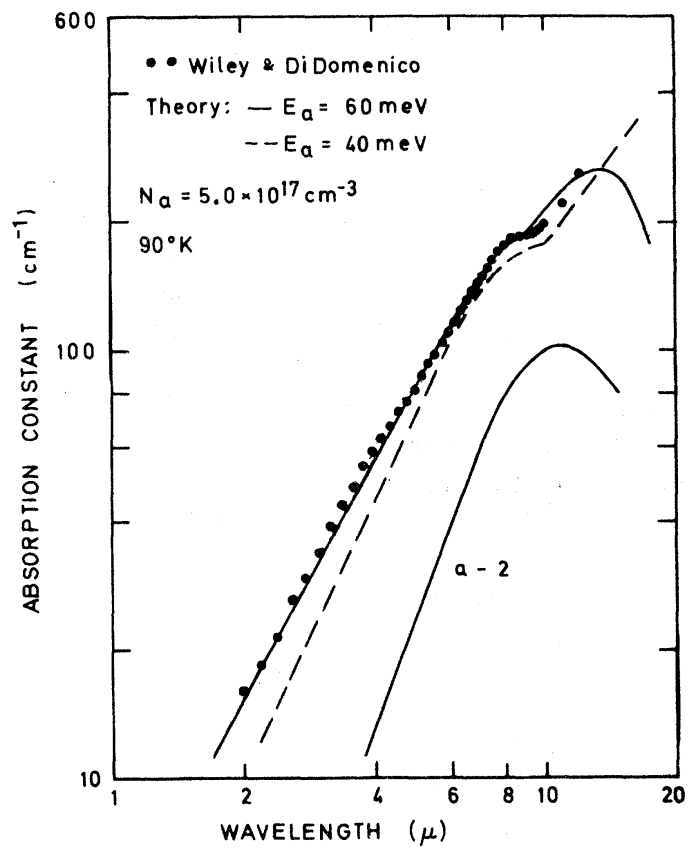

FIG. 6. Absorption constant of $p$-type GaP at $90^{\circ} \mathrm{K}$. Filled circles: experimental results of Ref. 3. Full line and broken line: calculated absorption from neutral acceptors with binding energies of 60 and $40 \mathrm{meV}$, respectively. Also shown separately is contribution from $a-2$ transitions $\left(E_{a}=60 \mathrm{meV}\right)$. The value of the anisotropy parameter $q$ is 1 in all calculations.

tribute to the absorption. The dashed line in Fig. 5 shows the contribution to the absorption from 2-3 transitions. This is most significant at large wavelenths and for $\lambda<4 \mu, 2-3$ transitions constitute only around $10 \%$ of the total absorption. The general agreement between calculated and experimental spectra is rather encouraging, the maximum deviation over the spectrum being $15 \%$. We have not taken into account any contribution from neutral acceptors here since the uncertainty intervals for the acceptor and hole concentrations overlap. WD have shown that the free-carrier absorption of the heavy-hole band might be enhanced owing to the existence of intermediate states in the light-hole and split-off band. The deviation between our calculated spectrum and the measured absorption at long wavelengths is in good agreement with WD's estimate of the intraband free-carrier absorption.

At $90^{\circ} \mathrm{K}$ only a negligible fraction of free holes is present. The experimentally observed absorption at this temperature (Fig. 6) is therefore due to absorption from neutral acceptors. The absorption bands for transitions to the different sublevels of the valence band overlap (see Fig. 2). It is therefore not feasible to use the simple theory in Sec. II B. The numerical computations 
of the absorption from neutral acceptors with the approximations of Eq. (18) gave best agreement with experimental data using $E_{a}=60 \mathrm{meV}$ and $q=1$. The experimentally determined binding energy of $\mathrm{Zn}$ acceptors in GaP is $60 \mathrm{meV}$ at low concentrations. ${ }^{19}$ Absorption spectra calculated with $q=0, E_{a}=60 \mathrm{meV}$ deviated $0-20 \%$ from the experimental values over the spectrum. This might, however, be due to the approximations of Eq. (18). The computed spectrum for $E_{a}=60 \mathrm{meV}$ and $q=1$ is shown in Fig. 6 together with the contribution from $a-2$ transitions. Also shown is the calculated absorption for $E_{a}=40 \mathrm{meV}, q=1$. The general agreement with $E_{a}=60 \mathrm{meV}, q=1$ is very good, both with respect to spectral shape and to absolute value.

Contrary to GaAs, there is no disagreement between experiment and theory at small wavelengths due to the influence of the core potential of the $\mathrm{Zn}$ ion and the local strain fields. The absorption at $2 \mu$ corresponds to $a-3$ transitions at wave vector $k=8 \times 10^{-2}$ a. u. , corresponding approximately to spatial distances of $10 \AA$. The first and second Bohr radii are 5 and $20 \AA$, respectively, using the heavy-hole mass of Table I. As for GaAs, the spatial extension of the core potential is then within the second Bohr radius.

\section{SUMMARY}

In this work we have presented a theory which quantitatively accounts for the absorption from neutral acceptors in GaAs and GaP. This absorption is due to transitions of bound holes from the acceptor state to the valence-band sublevels. It can be calculated from the bound-hole wave function and the optical matrix elements between the valence-band levels. The parameters of the calculation have been checked by calculating the absorption due to inter-valence-band transitions. Here, good agreement with experimental results was obtained. In the calculation of absorption from neutral acceptors, the adjustable parameters were the acceptor binding energy $E_{a}$ and the anisotropy parameter $q$. The values used for $E_{a}$ are in good agreement with recent experimantal values. Only a weak dependence on $q$ was observed.

The present model for absorption from neutral acceptors can be used in a study of acceptor wave functions. The bound-hole wave function is expanded in terms of Bloch functions of the valenceband levels. At any wave vector these expansion coefficients govern the absorption from transition of bound holes to all three valence-band levels. A computation of the absorption with a wave function slightly different from the actual one will therefore show disagreement with experiment in three regions of the spectrum (provided that all three regions are observable). As demonstrated for GaAs, this feature facilitates interpretation. We finally mention that whereas the inter-valenceband absorption can be partly superposed by absorption due to phonon-assisted processes, this effect should be small when all holes are frozen out.

\section{ACKNOWLEDGMENTS}

The author is grateful to Professor N. I. Meyer for his continuous support of this work and to the Northern Europe University Computing Center (NEUCC) for providing the computer time for the calculations presented here. During the course of this work, the author has benefitted greatly from discussions with Professor I. Balslev. Dr. J. D. Wiley kindly supplied the original data on the absorption in $p$-type GaP.

\footnotetext{
*Present address: IBM Watson Research Center, Yorktown Heights, N. Y。 10598.

${ }^{1}$ R. Braunstein, J. Phys. Chem. Solids 8, 280 (1959).

${ }^{2}$ I. Balslev, Phys. Rev. 177, 1173 (1969)。

${ }^{3}$ J. D. Wiley and M. DiDomenico, Jr., Phys. Rev。B 3, 375 (1971).

${ }^{4}$ A. H. Kahn, Phys. Rev. 97, 1647 (1955).

${ }^{5}$ R. Newman and W. W. Tyler, Phys. Rev. 105, 885 (1957).

${ }^{6}$ W. G. Spitzer and H. Y. Fan, Phys. Rev, 106, 882 (1957).

${ }^{7}$ J. B. Arthur, A. C. Baynham, W. Fawcett, and E. G. S. Paige, Phys. Rev. 152, 740 (1966).

${ }^{8} \mathrm{I}$. Balslev and $\mathrm{O}$ 。Christensen (unpublished).

${ }^{9}$ T. P. McLean, in Progress in Semiconductors, edited

by A. F. Gibson (Heywood, London, 1960), Vol. 5.

${ }^{10}$ D. M. Eagles, J. Phys. Chem. Solids 16, 76 (1960).
}

\footnotetext{
${ }^{11} \mathrm{~J}$. M. Luttinger and W. Kohn, Phys. Rev。 $\underline{97}, 869$ (1955)。

${ }^{12}$ W. Fawcett, Proc. Phys. Soc. (London) 85, 831 (1965)。

${ }^{13}$ G. Dresselhaus, A. F. Kip, and C. Kittel, Phys. Rev. 98, 368 (1955).

${ }^{14} \mathrm{E}$. O. Kane, J. Phys. Chem. Solids 1,82 (1956).

${ }^{15} \mathrm{~A}$. C. Baynham and E. G. S. Paige, in Proceedings of the Seventh International Conference on the Physics of Semiconductors (Academic, New York, 1964), p. 149.

${ }^{16}$ P. Lawaetz, Phys. Rev. B $\underline{4}, 3460$ (1971).

${ }^{17}$ D. E. Hill, J. Appl. Phys. 41,1815 (1970); Phys。 Rev. B 1, 1863 (1970).

${ }^{18}$ G. Lucovsky, Solid State Commun。 3, 299 (1965).

${ }^{19} \mathrm{H}$. C. Casey, F. Ermanis, and K. B. Wolfstirn, J. Appl. Phys. 40, 2945 (1968).
} 